\title{
Acerca del carácter irreductible de la mens humana en Nicolás de Cusa: unidad y número*
}

Claudia D'Amico"

CONICET. Universidad de Buenos Aires

Argentina

Para citar este artículo: D'Amico, Claudia. «Acerca del carácter irreductible de la mens humana en Nicolás de Cusa: unidad y número».

Franciscanum 169, Vol. Lx (2018): 87-107.

\section{Resumen}

Nicolás de Cusa elabora la doctrina del espíritu humano como una Metafísica de la Subjetividad. El Cusano muestra que el ser de la mente es su propia operación y su propio despliegue se constituye en forma o entidad del mundo conjetural. El despliegue de cada mente en un mundo simbólico tiene un carácter singular e irreductible. Para desarrollar esta doctrina que preanuncia la modernidad, el Cusano atiende a la relación entre unidad y número procedente de la tradición platónico-pitagórica.

Este trabajo incorpora resultados presentados en el workshop internacional Irreductibilidad y persona realizado en Santiago de Chile (2016), en el marco del proyecto de investigación interdisciplinar Singularidad. Convergencia de filosofía, teología y ciencia de la Universidad Católica del Maule, financiado por Templeton Foundation y adjudicado por el Proyecto Science, Philosophy and Theology: Capability Building in Latin America» de la Universidad de Oxford.

Doctora en Filosofía por la Universidad de Buenos Aires, profesora titular de filosofía medieval de la Universidad Nacional de la Plata, profesora asociada de filosofía medieval de la Universidad de Buenos Aires e investigadora principal del CONICET. Es codirectora del Círculo de Estudios Cusanos de Buenos Aires y vicepresidenta de la Sociedad Iberoamericana de Neoplatonismo. Investiga el pensamiento de Nicolás de Cusa y sus fuentes neoplatónicas. Contacto: claudiadamico@yahoo.com.ar. 


\title{
Palabras clave
}

Nicolás de Cusa, mente, número, unidad, subjetividad.

\section{About the irreducible character of the humana mens in Nicholas of Cusa: Unity and number}

\begin{abstract}
Nicholas of Cusa elaborates the doctrine of the human spirit as a Metaphysics of Subjectivity. The Cusanus shows that the being of mind is its own operation and its own deployment that becomes either a form or a conjectural world entity. The deployment of every mind in a symbolic world has a unique and irreducible character. In order to elaborate this doctrine that advances modernity, the Cusanus responds to the relation between unit and number, which comes from the Platonic-Pythagorean Tradition.
\end{abstract}

\section{Keywords}

Nicholas of Cusa, Mind, Number, Unity, Subjectivity.

Es bien sabido que la figura de Nicolás de Cusa comenzó a ser decisiva en la historiografía del siglo $\mathrm{xx}$, a partir de los estudios de Ernst Cassirer ${ }^{1}$. Actualmente, ya no solo se realiza esta referencia

1 Cf. Ernst Cassirer, Das Erkenntnisproblem in der Philosophie und Wissenschaft der neueren Zeit (Berlín: Cassirer, 1906). Edición castellana: El problema del conocimiento en la filosofía y en la ciencia modernas (México: FCE, 1979); Individuum und Kosmos in der Philosophie der HYPERLINK https://es.wikipedia.org/wiki/Renacimiento lo Renacimiento Renaissance (Leipzig: Teubner, 1927). Edición castellana: Individuo y cosmos en la filosofía del Renacimiento (Buenos Aires: EMECÉ, 1951). 
insoslayable, sino que un reciente trabajo analiza filosóficamente esta cuestión trascendiendo los límites de un rescate meramente historiográfico ${ }^{2}$. En efecto, la gravitación de su pensamiento en Alemania en los siglos XIX y xx, muy especialmente, en los filósofos neokantianos, se debe al hecho de que el Cusano puede tomar partido en una cuestión que presenta para ellos una alternativa: el problema de la Subjetividad o el problema de la Individualidad ${ }^{3}$. En esta disyunción, los filósofos de Marburgo destacan el concepto de Subjetividad ligando al Cusano con la Modernidad, más que con sus fuentes medievales. Rudolf Eucken, Richard Falckenberg aun antes de Hermann Cohen y el propio Cassirer, se ocuparon del pensamiento cusano buscando un lugar privilegiado en la historia de la filosofía, entre Platón y Kant, para su «Geisttheorie».

Desde entonces y, hasta hoy, muchos estudiosos proponen para el Cusano un rol decisivo en lo que se conoce como «metafísica del espíritu», ampliando estos límites y trazando muchas veces una línea desde el platonismo hasta el idealismo alemán ${ }^{4}$. Incluso en la dicotomía entre henología o pensamiento de lo uno y ontología o pensamiento del ser, la filosofía cusana se ubicaría como una reflexión sobre el ser o lo uno, acompañada por una teoría del intelecto solo a través del cual el ser o lo uno pueden ser pensados ${ }^{5}$. La clave de la distinción sería entonces, sobre todo, una teoría del espíritu -tanto divino como humano-, que compromete toda henología y toda ontología.

La doctrina cusana sobre el espíritu divino o divina mens hace especial hincapié en la unidad-trinidad de este principio intelectual de

2 Cf. Kirstin Zeyer, Cusanus in Marburg. Hermann Cohens und Ernst Cassirers produktive Form der Philosophiegeschichtsaneignung (Münster: Aschendorff, 2015).

3 Cf. Huber Benz, Individualität und Subjektivität. Interpretationstendenzen in der Cusanus-Forschung und das Selbstverständnis des Nikolaus von Kues (Münster: Aschendorff, 1999).

4 Sin duda, en este sentido, son fundamentales los estudios de Werner Beierwaltes. Citaremos solo dos: el fundacional Platonismus und Idealismus (Frankfurt am Main: Klostermann, 1972); Identität und Differenz, (Frankfurt am Main: Klostermann, 1980).

5 Cf., por ejemplo, Karl-Heinz Volkmann-Schulck, Nikolaus Cusanus. Die Philosophie im Übergang vom Mittelalter zur Neuzeit (Frankfurt am Main: Klostermann, 1957); Josef Stallmach, «Die cusanische Erkenntnisauffassung zwischen Realismus und Idealismus», Mitteilungen und Forschungsbeiträge der Cusanus-Gesellschaft Vol. 6 (1967): 50-54. 
todas las cosas, sin que esta conformación trinitaria afecte en nada su absoluta simplicidad. Si atendemos a la doctrina del espíritu humano o humana mens cusana, también en ella se realza una constitución unitrina; sin embargo, tal operar uni-trinitario está indisolublemente ligado al número. Analizando estas nociones procuraremos mostrar cómo la metafísica de Nicolás de Cusa, al tematizar el espíritu humano, no lo hace desde la perspectiva de una especie de subjetividad trascendental sino que, por el contrario, su análisis destaca el carácter irreductible y, a la vez, único de cada mente humana. Así, su filosofía del espíritu debe ser, necesariamente, vinculada a la metafísica de la singularidad, que orienta todo su pensamiento ${ }^{6}$. Si para el Cusano, cada singular despliega de modo peculiarísimo la infinita unidad de Dios, concebida como "Singularidad de las singularidades», cada espíritu humano, muy particularmente, lo despliega de un modo dinámico u operativo. De esta manera, su rol de precursor de la metafísica de la subjetividad debe ser, necesariamente, redefinido en términos de singularidad e irreductibilidad ${ }^{7}$.

\section{II}

Las nociones de unidad y número tienen, en la filosofía cusana, un sentido matemático, pero también, y fundamentalmente, un sentido metafísico. La unidad es sinónimo de identidad y, en tal sentido, es entendida como indivisibilidad e irreductibilidad.

El número se entiende como despliegue de la unidad, su explicitación. Cada número es una aparición de la unidad en la multiplicidad, la unidad en todo número se presenta como lo irreductible. Desde esta perspectiva, ambas nociones se vinculan a una tradición filosófica: el platonismo y sus raíces pitagóricas. Sin duda, la obra de Nicolás de Cusa es, en cierto sentido, una versión

6 Cf. Rodrigo Nuñez Poblete, Metafísica de la singularidad. La noción de singularitas en la filosofía de Nicolás de Cusa (Buenos Aires: Biblos, 2015).

$7 \quad$ Acerca de los perfiles de esta discusión, cf. Harald Schwaetzer, «Nikolaus von Kues als Vordenker der Subjektivität», en Zum Subjektbegriff bei Meister Eckhart und Nikolaus von Kues, eds. Harald Schwaetzer, Marie-Anne Vannier (Münster: Aschendorff, 2011), 67-75. 
tardía y cristianizada de esta tradición, en la cual, estas nociones se desplazan desde el plano de lo real al ámbito de lo humano, fundamentalmente, al del despliegue cognoscitivo ${ }^{8}$.

En su primera gran obra filosófico-teológica, De docta ignorantia de 1440, el Cusano, después de mostrar que en lo máximo absoluto los opuestos coinciden -lo que se conoce como la célebre coincidentia oppositorum cusana- refiere que tal máximo absoluto es lo uno simplísimo y relaciona toda discreción con el número. Desde esta primera presentación atiende a la doctrina pitagórica, según la cual, el número está compuesto de identidad y alteridad. Esta doctrina pudo ser leída por el Cusano en muchos textos de la tradición, entre los cuales se destaca, sin duda, De institutione arithmetica de Boecio y sus desarrollos acerca de la magnitud y la multitud, cuya fuente es el pitagórico Nicómaco de Gerasa9 ${ }^{9}$. Conviene revisar una larga cita que es, de algún modo, fundacional del pensamiento cusano ${ }^{10}$ :

Y porque todas las cosas son de aquel modo mejor que el que pueden ser, entonces no puede haber pluralidad de entes sin el número. Pues suprimiendo el número cesan la distinción, el orden, la proporción, la armonía de las cosas y, además, la misma pluralidad de los entes [...], es claro que el ascenso del número es finito en acto y que tal número estará en potencia para otro. Y si en el descenso el número se comportara de la misma manera, que dado cualquier número pequeño en acto -puesto que, entonces, siempre podría darse, por sustracción, uno menor, como en el ascenso por adición se da uno mayor-, aun así sería lo mismo, porque no habría ninguna distinción de las cosas, y no se encontraría en los números ni orden, ni pluralidad, ni lo que excede ni lo excedido, aún más: no habría número. Por lo cual es necesario que en el número se llegue a lo mínimo, cuyo menor no puede haber, tal como es la unidad. Y puesto que para la unidad no puede haber menor, la unidad será lo

8 Cf. Inigo Bocken, «Die Zahl als Gründlage der Bedeutung bei Nikolaus von Kues», Mitteilungen und Forschungsbeiträge der Cusanus-Gesellschaft Vol. 29 (2005): 210-213; Anke Eisenkopf, Zahl und Erkenntnis bei Nikolaus von Kues (Regensburg: Roderer-Verlag, 2007), 9-13.

9 Cf. Cecilia Rusconi, El uso simbólico de las figuras matemáticas en la metafísica de Nicolás de Cusa (1401-1464) (Buenos Aires: Biblos, 2012), 161-165.

10 Las obras cusanas se citan de acuerdo a la edición crítica: Nicolai de Cusa, Opera Omnia, Iussu et Auctoritate Academiae Litterarum Heidelbergensis, Leipzig, 1932 ss., indicando, en cada caso, el volumen y número de parágrafo. 
mínimo en cuanto tal, lo que coincide con lo máximo, según lo mostrado recientemente ${ }^{11}$.

Si lo máximo es la unidad, lo mínimo, también lo es: ambos coinciden entendidos como absolutos. Ahora, entre los extremos de la coincidente unidad, se da el territorio del más y del menos, del número que explica la pluralidad. En la serie enumerada, la unidad máxima-mínima, a la vez, se explicita o despliega a sí misma, diversamente. $\mathrm{Si}$, como afirma el Cusano, «conocer es establecer proporciones», razón por la cual, la coincidencia de máximo y mínimo, en cuanto improporcionable, resulta incognoscible, tal proporción no cuenta con otro instrumento que el número: cada número es idéntico a sí mismo, pero, diverso de otro y esto lo vuelve comparable. Claramente, la descripción de la pluralidad no puede entenderse separada de la operación de un espíritu humano; en otras palabras, el Cusano no piensa en una realidad conformada numéricamente o escrita por Dios en caracteres matemáticos, sino que piensa en un despliegue de la unidad absoluta que es numerada por el hombre.

Esto se explicita muy claramente, en De coniecturis, obra que el Cusano comienza a escribir, conjuntamente, con De docta ignorantia, pero culmina algunos años después. Afirma allí algo revelador, que esclarece lo dicho sobre la proporción: el número es el primer ejemplar de la mente. Si esto es así, la misma multiplicidad parece provenir

11 De doct. ign. (h I) n.13: «Et quoniam omnia sunt eo meliori modo, quo esse possunt, tunc sine numero pluralitas entium esse nequit; sublato enim numero cessant rerum discretio, ordo, proportio, harmonia atque ipsa entium pluralitas. Quod si numerus ipse esset infinitus - quoniam tunc maximus actu, cum quo coincideret minimum -, partier cessarent omnia praemissa. In idem enim redit numerum infinitum esse et minime esse. Si igitur ascendendo in numeris devenitur actu ad maximum, quoniam finitus est numerus: non devenitur tamen ad maximum, quo maior esse non possit, quoniam hic foret infinitus. Quare manifestum est ascensum numeri esse finitum actu et illum in potentia fore ad alium. Et si in descensu pariter se numerus haberet, ut dato quocumque parvo numero actu, quod tunc per subtractionem semper dabilis esset minor sicut in ascensu per additionem maior, - adhuc idem; quoniam nulla rerum discretion foret, neque ordo neque pluralitas neque excedens et excessum in numeris reperiretur, immo non esset numerus. Qua propter necessarium est in numero ad minimum deveniri, quo minus esse nequit, uti est unitas. Et quoniam unitati minus esse nequit, erit unitas minimum simpliciter, quod cum maximo coincidit per statim ostensa.» Nicolás de Cusa, Acerca de la docta ignorancia. Libro primero, Introducción, traducción y notas Jorge Mario Machetta y Claudia D'Amico (Buenos Aires, Biblos, 2003). 
de la mente, o al menos la multiplicidad en cuanto proporcionable. Todas las recurrencias aritméticas que presenta la obra, comenzando por la tetraktys pitagórica, deben ser entendidas en este sentido: son símbolos que crea la mente humana para representar la estructura de lo real: 1, 10, 100, 1000, representan, respectivamente, la unidad divina, la intelectual, la anímica y la corpórea ${ }^{12}$. Asimismo, cuando explicita el modo en que la unidad de la mente progresa desde sí misma para retornar a sí, atiende al sentido simbólico de los números seis, siete y diez como un camino para develar, de algún modo, la progresión natural ${ }^{13}$. El desplazamiento hacia el espíritu humano se ha producido ${ }^{14}$.

\section{III}

Más adelante, en el marco de los diálogos, cuyo personaje principal es el Idiota o ignorante, se aborda en particular el tema de la mente. El término mens, de larga tradición medieval, es deliberadamente ofrecido para titular el escrito, diferenciándolo del de anima. En el comienzo del diálogo entre el orador, el filósofo y el idiota, se establece la siguiente distinción, que es necesario poner de relieve:

Ig: [...] la mente, es aquello desde lo cual se da el término y medida de todas las cosas. Pues interpreto que mente, ciertamente, se dice por el medir.

Fil: ¿̇Piensas que una cosa es la mente y otra el alma?

Ig: Ciertamente lo pienso. Pues una es la mente que subsiste en sí misma, otra la que está en un cuerpo. La mente subsistente en sí misma o es infinita o es imagen de lo infinito. De aquellas, sin embargo, que son imagen de lo infinito, puesto que no son máximas o absolutas, es decir

12 Cf. De coni. (h III) n. 10-11.

13 Cf. De coni. (h III) n. 106.

14 Cf. Klaus Kremer, «Grösse und Grenze der menschlichen Vernunft», en Nicholas of Cusa. A Medieval Thinker for the Modern Age, ed. Kazuhiko Yamaki (Waseda: Curzón, 2002), 5-34. 
infinitas en sí subsistentes, admito que pueden algunas animar al cuerpo humano y entonces, por oficio, concedo que son almas ${ }^{15}$.

La elección del término mens pone de manifiesto acaso una decisión cusana de diferenciar su abordaje del de los frecuentes tratados De anima o de los comentarios al texto aristotélico para incorporarlo a otra tradición, la de los tratados De intellectu ${ }^{16}$. De hecho, luego de esta distinción el término anima no volverá a aparecer en el diálogo y se hablará solo de mens o intellectus. Esta apuesta no es del todo original. Es necesario considerar antecedentes inmediatos que provienen de la llamada Escuela albertista de Colonia de los siglos XIII y XIV. Autores como Dietrich de Freiberg, sucesor de Alberto, por ofrecer solo un ejemplo, muestran que, lejos de constituir una sustancia, la identidad del intelecto es una identidad activa, cuyo ser es operar ${ }^{17}$. Sin embargo, esta doctrina cobrará en el Cusano matices particulares que intentaré subrayar.

En el texto citado de De mente se afirma: (1) por una parte, que este vocablo mens es aplicable o bien, en sentido absoluto, infinito, es decir a Dios, o bien, como imagen de lo infinito, esto es al hombre; (2) por otra, que el vocablo mens, vinculado a mensurare, es aquello desde lo cual se da término y medida a todo; (3) por último, se concede que la mens, que es imagen de lo infinito, se la llame anima ex officio mientras esté unida al cuerpo. Este breve texto, pues, compendia estos tres tópicos, fundamentales, respecto del tratamiento cusano del tema, en estas y otras obras anteriores y posteriores.

15 De mente ( $\mathrm{h}$ 2) n. 57: «Idiota: Puto neminem esse aut fuisse hominem perfectum, qui non de mente aliqualem saltem fecerit conceptum. Habeo quidem et ego: mentem esse, ex qua omnium rerum terminus et mensura. Mentem quidem a mensurando dici conicio. Philosophus: Putas ne aliud mentem, aliud animam? Idiota: Puto certe. Nam alia est mens in se subsistens, alia in corpore. Mens in se subsistens aut infinita est aut infiniti imago. Harum autem, quae sunt infiniti imago, cum non sint maximae et absolutae seu infinitae in se subsistentes, posse aliquas anima re humanum corpus admitto, atque tunc ex officio easdem animas esse concede». Nicolás de Cusa, Un ignorante discurre acerca de la mente. Idiota. De mente. Edición bilingüe, trad. Jorge Mario Machetta, notas Círculo de Estudios Cusanos del Buenos Aires (Buenos Aires: Biblos, 2005).

16 Cf. Jorge Mario Machetta, «Introducción» en Nicolás de Cusa. Un ignorante discurre acerca de la mente. Idiota de mente, 9-20.

17 Cf. Kurt Flasch, «Einleitung» en Opera Omnia, Dietrich von Freiberg (Hamburg: Meiner, 1977), p. XI 
En De docta ignorantia, el Cusano había planteado una imagen que ilustra la actividad del intelecto en relación con la verdad. Dice que nuestro intelecto se dirige a la verdad, como el polígono inscripto en un círculo que, por más que multiplique sus ángulos, no podrá alcanzarlo nunca ${ }^{18}$. De allí, que no accedamos a la verdad en sí, sino en tanto, dada en lo múltiple. Esta imagen preanuncia la definición cusana de conjetura, como aserción positiva de la verdad, en la alteridad que ofrecerá en De coniecturis ${ }^{19}$. En este escrito, Nicolás de Cusa plantea por primera vez, un paralelo entre la mente divina y la mente humana, que será en adelante, la clave de todo su planteo onto-gnoseológico. Allí afirma: «Conviene que las conjeturas provengan de nuestra mente, tal como el mundo real proviene de la infinita razón divina» ${ }^{20}$.

Así como de la razón o intelecto divino proviene el mundo, como su propia explicitación o despliegue, del mismo modo, la mente humana se explicita a sí misma en su propio mundo, el mundo que el cusano llama conjetural. Continúa diciendo:

Por lo tanto, la mente humana es forma conjetural del mundo tal como la divina del real. Por lo cual, así como aquella absoluta divina entidad es todo aquello que es, en todo lo que es, de la misma manera, también, la unidad de la mente humana es la entidad de sus conjeturas ${ }^{21}$.

Es muy interesante advertir que, Nicolás de Cusa, confiere al mundo conjetural dos atribuciones propias del mundo real: forma y entitas. Forma y entidad son otorgadas por la unidad de la mente humana que se explicita a sí misma, en un mundo que es completamente suyo.

Sin duda, el fundamento del paralelismo es, en última instancia, teológico y se desprende de la sentencia escrituraria del

18 Cf. De docta ignorantia (h I) n. 10.

19 Cf. De coni. (h III) n. 2.

20 De coni. (h III) n.5: «Coniecturas a mente nostra, uti realis mundus a divina infinita ratione, pro dire oportet». (Traducción inédita Jorge Mario Machetta).

21 De coni. (h III) n.5: «Coniecturalis itaque mundi humana mens forma exstitit uti realis divina. Quapropter ut absoluta illa divina entitas est omne id quod est in quolibet quod est, ita et mentis humanae unitas est coniecturarum suarum entitas». 
hombre, como imago dei. Con todo, a la hora de precisar en qué aspecto son semejantes el ejemplar, Dios, y su imagen, el hombre, el Cusano no duda en afirmar que es, precisamente, por la posibilidad de su propia explicitación que reside en su carácter intelectual:

Ahora bien, Dios obra todo por causa de sí mismo, de modo que sea a la vez principio intelectual y fin de todo; de la misma manera, ciertamente, la explicación racional del mundo proveniente de nuestra mente complicante que es hacedora por causa de sí misma ${ }^{22}$.

Precisamente, si algo es propio del intelecto infinito y, también, de su imagen es su carácter productivo y esto no es debido a ningún otro más que a sí mismo, incluso la mente o intelecto humano, igualmente, produce solo por causa de sí mismo. El movimiento que define, en primer lugar, a ambos intelectos es el reflexivo: el intelecto se piensa a sí mismo, el entendimiento de sí es fundante. Sin embargo, en este mismo movimiento de los intelectos, divino y humano hay, por una parte, una completa desemejanza y, al mismo tiempo, un rasgo semejante.

Del lado de la desemejanza, la actividad del intelecto divino tiene un movimiento ad intra y otro ad extra. El pensarse a sí mismo del divino intelecto es la interna auto-constitución trinitaria y tal movimiento produce hacia fuera, el universo entendido como explicatio dei. El pensarse a sí mismo del intelecto humano, en cambio, produce solo ad extra. El intelecto humano se explicita a sí mismo, solo en la alteridad pensada; en este sentido, el mundo conjetural es concebido como explicatio mentis. Precisamente, que la reflexión humana sea despliegue en la alteridad, implica que su unidad produzca el número como primer ejemplar. Es notable que el Cusano lo denomine ejemplar simbólico ${ }^{23}$. Con esto quiere destacar, por una parte, que el número ofrece un modo adecuado de referirse pariter et finis omnium; ita quidem rationalis mundi explicatio, a nostra complicante mente progrediens, propter ipsam est fabricatricem».

23 Este es precisamente el título del capítulo 2 de la obra: «Symbolicum exemplar rerum numerum esse». Cf. De coni. (h III) n. 7. 
a la pluralidad en tanto, siguiendo la tradición boeciana, considera que el número es lo único compuesto de sí mismo; por otra, que este modo de referencia es puramente un producto humano: el número es solo razón humana explicitada ${ }^{24}$.

Del lado de la semejanza: ambos intelectos realizan una actividad cuyo movimiento es trino $y$, por esto mismo, es necesariamente fecunda. Si se busca la similitud en su producción en la alteridad, es decir en lo otro de sí mismos, esta actividad es definida como aquella que pone término y medida, por esto ambos pueden ser llamados «mentes»: en tanto la mente infinita es la Medida de todo puesto que pone límite ontológico a todo lo que es su explicatio sin ser medida por nada; la mente humana es medida conjetural o nocional de todo siendo solo medida por aquella, la mente infinita, de la cual es imagen ${ }^{25}$.

Ahora bien, en De mente no solo se trata de la mens en cuanto tal sino que se trata de lo que ella es ex officio, es decir, en cuanto alma que anima un cuerpo. El idiota señala una y otra vez el hecho de que la mente ha sido creada en el cuerpo y de que tiene necesidad de él. Cito:

La fuerza de la mente, que es la fuerza comprehensiva de las cosas y la fuerza nocional, no puede actuar en sus operaciones sino es excitada por lo sensible [...]. Por lo tanto necesita del cuerpo orgánico pues sin él no podría realizarse la excitación ${ }^{26}$.

El cuerpo liga al hombre al mundo y el mundo señala al hombre una tarea: solo la mente humana, inserta en la vida corpórea, puede participar su naturaleza de imago al resto de las creaturas. De hecho, afirma, en un llamativo pasaje:

24 De coni (h III) n. 7: «Nec est aliud numerus quam ratio explicata».

25 Cf. Michael Stadler, «Zum Begriff der mensuratio bei Cusanus. Ein Beitrag zur Ortung der cusanischen Erkenntnislehre», Miscelania Mediaevalia Vol. 16 (1983): 118-131.

26 De mente ( $\mathrm{h}$ 2 ) n. 77: «sic vis mentis, quae est vis comprehensiva rerum et notionalis, non potest in suas operationes, nisi excitetur a sensibilibus [...]. Opus ergo habet corpus organico, tali scilicet, sine quo excitatio fieri non potest». 
De donde en cuanto todo después de la simple mente participa de la mente, en tanto participa también de la imagen de Dios, de modo que la mente sea de por sí imagen de Dios y todo lo que es después de la mente no sea sino por medio de la mente ${ }^{27}$.

Cuando el hombre conoce, y subrayemos aquí cuando cada hombre conoce, participa su naturaleza de imagen a todos los demás aspectos del universo que carecen de mente. La naturaleza de imagen pertenece a todos por igual, pero la manera en que la participa es singularísima.

Esta participación de su naturaleza mental es ante todo un hacer visible, un dejar ver a través de sí su propia unidad y la unidad de la mente divina. El ignorante parece querer señalar que como imago, la mente humana sirve a la manifestación divina, completándola. Esta tarea se realiza mediante la asimilación nocional del mundo. Según enseña el idiota:

[...] haciendo nociones o visiones intelectuales: la mente divina es fuerza entificativa, nuestra mente es fuerza asimilativa ${ }^{28}$.

La mente no es mera imago dei sino viva imago dei, no es una sustancia como substrato de las pasiones, sino que es una viva substantia, cuya esencia no es otra que su propia actividad asimilativa ${ }^{29}$.

La vida de la que se habla no es otra que la vida intelectual -de la que el resto de los seres carecen- y que, una vez despierta y puesta en movimiento por la admiración de las cosas mundanas, mueve constantemente la mente hacia el ejemplar de todas las cosas, del cual ella misma es imagen. En la medida en que en la humana mens reluce propiamente el ejemplar, ella puede encontrar en sí misma lo que busca. Con todo, es necesario llamar la atención que

27 De mente ( $\mathrm{h} \vee 2)$ n. 73: «Unde quantum omnes res post simplicem mentem de mente participant, tantum et de dei imagine, ut mens sit per se dei imago et omnia post mentem non nisi per mentem». Subrayado mío.

28 De mente ( $\mathrm{h}$ v 2) n. 99: «Divina mens concipiendo creat, nostra concipiendo assimilat notiones seu intelectuales faciendo visiones. Divina mens est vis entificativa, nostra mens est vis assimilative».

29 Cf. Gerda von Bredow, «Der Geist als lebendiges Bild gottes (Mens viva dei imago)», Mitteilungen und Forschungsbeiträge der Cusanus-Gesellschaft Vol.13 (1978): 58-67. 
este «ejemplar» no es entendido como un contenido apriorístico sino meramente como la actividad perfecta de un intelecto productivo ${ }^{30}$.

La vida de la mente solo se excita a partir de la admiración originada por las cosas sensibles. Por eso necesita del cuerpo orgánico que habita y que anima. Ella es medida viviente. Recorre lo sensible, midiendo para conocer $y$, sobre todo, para conocerse a sí misma:

Pues la mente es medida viviente, que midiendo otras cosas alcanza la propia capacidad. Pues todo lo hace para conocerse a sí misma. Pero no encuentra la medida de sí buscando en todas las cosas, sino donde todas ellas son uno. Allí está la verdad de su precisión, porque allí está su ejemplar adecuado ${ }^{31}$.

El conocimiento del mundo, puesto que está siempre mediado por la unidad de la mente y su despliegue, constituye el primer paso para el conocimiento de sí. Siendo la viva imagen de Dios, la mente se encuentra en la paradójica condición de poder trascenderse siempre en dirección al ejemplar divino, con el que sin embargo no coincidirá nunca justamente por ser solo su imagen. La ejemplificación del polígono inscripto en el círculo, que mencionamos anteriormente, representa esta dinámica inagotable de la mente humana. El número siempre en potencia hacia uno mayor o menor, en busca de la coincidencia, en busca de su unidad.

Como viva imagen de la mente del creador, la humana mens conserva e involucra en su operar un modo esencialmente creativo. No es azaroso que el personaje central del diálogo, el idiota, sea en este caso un artesano, un hacedor de cucharas, ni que sus enseñanzas sobre la mente y los ejemplos con los cuales las ilustra nazcan a partir de la «investigación simbólica» consumada en su arte. La mente humana puede ser llamada con propiedad imago dei en tanto creadora: de las formas de los entes artificiales (como la cuchara) y de

30 Cf. Klaus Kremer, «Erkennen bei Nikolaus von Kues. Apriorismus - Assimilation - Abstraktion», Mitteilungen und Forschungsbeiträge der Cusanus-Gesellschaft Vol. 13 (1978): 23-57.

31 De mente ( $\mathrm{v}$ 2) n. 123: «Nam mens est viva mensura, quae mensurando alia sui capacitatem attingit. Omnia enim agit, ut se cognoscat. Sed sui mensuram in omnibus quaerens non invenit, nisi ubi sunt omnia unum. Ibi est veritas praecisionis eius, quia ibi exemplar suum adaequatum». 
entia rationis entre los que se incluyen los entes matemáticos y todo nombre en general, incluso los géneros y las especies. La posibilidad de crear formas artificiales será retomada por Nicolás en De Beryllo de 1458 y lo conducirá a llamar al hombre secundus deus, recreando de una manera peculiar la fórmula hermética ${ }^{32}$. Es necesario destacar que más allá de que se atienda a los entes matemáticos como una clase específica de creación humana, toda actividad mental, aún la creación de formas artificiales, es numérica.

Sin embargo, en De mente, se insiste particularmente en la creación de nociones siempre en paralelo con la mente divina:

La concepción de la mente divina es la producción de las cosas; la concepción de nuestra mente es la noción de las cosas. Si la mente divina es entidad absoluta, entonces su concepción es la creación de los entes y la concepción de nuestra mente es la asimilación de los entes. Pues lo que conviene a la mente divina en cuanto infinita verdad, a nuestra mente conviene como la imagen próxima de aquella. Si todo está en la mente divina como en su precisa y propia verdad, todo está en nuestra mente como en la imagen o similitud de la propia verdad, esto es, nocionalmente: pues el conocimiento se realiza con la similitud ${ }^{33}$.

Los términos imago unitatis e imago complicationis, con los cuales Nicolás designa indistintamente a la mente, cobran sentido sobre todo en el contexto de la cuestión del conocimiento concebido como el arte de la creación de nociones. La mente posee la posibilidad de conocer la realidad complicándola notionaliter. Ella misma constituye el ámbito de complexión en el que las formas de todas las cosas se reúnen, ya no como mera multiplicidad, no como una pluralidad de ideas o razones, sino bajo la identidad de la unidad.

La mente usa de sí misma con este altísimo modo en cuanto ella misma es imagen de Dios; y Dios, quien es todo, reluce en ella. Es decir: cuando en

32 Cf. Claudia D'Amico, «Elementos neoplatónicos y herméticos en la concepción del hombre de Nicolás de Cusa» en La concepción del hombre en Nicolás de Cusa. Fuentes, originalidad y diálogo con la modernidad (Buenos Aires: Biblos, 2015), 81-92.

33 De mente ( $\mathrm{h}$ v 2) n. 72: «Conceptio divinae mentis est rerum productio; conceptio nostrae mentis est rerum notio. Si mens divina est absoluta entitas, tunc eius conceptio est entium creatio, et nostrae mentis conceptio est entium assimilatio. Quae enim divinae menti ut infinitae conveniunt veritati, nostrae conveniunt menti ut propinquae eius imagini. Si omnia sunt in mente divina ut in sua praecisa et propia veritate, omnia sunt in mente nostra ut in imagine seu similitudine propriae veritatis». 
tanto imagen viviente de Dios se vuelve, en el asimilar, con todo empeño hacia su ejemplar. Y de esta manera intuye que todo es uno y que ella es la asimilación de aquel uno, y a través de esta hace las nociones acerca de lo uno, que es todas las $\operatorname{cosas}^{34}$.

Cuando la mente conoce, la Unidad se hace presente en la imagen y a través de ella se pone de relieve la unidad del resto de los seres que no son capaces de conocimiento, ni de autoconocimiento. Toda actividad cognoscitiva, incluso la más elemental, conlleva la introducción de la unidad. No tendríamos la sensación de color si el aspecto activo de la mente no lo identificara como tal. La unidad de la mente está presente, como discernimiento en la multiplicidad, durante todas las instancias cognoscitivas, haciéndolas posibles. Por tanto incluso el grado de conocimiento más inmediatamente ligado a la experiencia material, la percepción sensible, no es puramente pasivo sino que, por lo contrario, depende de la actividad de la mente.

Para el hombre no hay mundo sin más, sino solo mundo asimilado a sí, mundo pensado con la unidad de la mente ${ }^{35}$. Sin embargo, la asimilación se realiza no por lo que ofrece la experiencia sino por la posibilidad de volverse sobre sí mismo, la recurrencia a lo sensible como estímulo aparece solo porque el intelecto como alma, ex officio, anima un cuerpo. El estímulo sensible hace que ella se vuelva reflexivamente a sí misma, se descubra como intelecto, y desde sí pueda producir nociones asimilativas del mundo. El movimiento, pues, no va desde la realidad sensible hacia el intelecto sino que parte del intelecto para volver a él.

\section{IV}

La mente humana es movimiento. En ese movimiento reside su semejanza con el primer principio: su movimiento también productivo es unitrino y en la medida en que la mente se va independizando del

\footnotetext{
34 De mente ( $\mathrm{h}$ 2) n. 106: «Utitur autem hoc altissimo mens se ipsa, ut ipsa est dei imago, et deus, qui est omnia, in ea relucet, scilicet quando ut viva imago dei ad exemplar suum se omni conatu asimilando convertit. Et hoc modo intuetur omnia unum illius unius assimilationem, per quam notiones facit de uno quod omnia». Cf. De mente (h v 2) n. 76.
} 
cuerpo, se reconoce como autosubsistente, se piensa a sí misma, y como su creador se reconoce como unitrina y libre. Irreductibilidad y libertad se vinculan.

Esto es presentado muy claramente por Nicolás de Cusa en una de sus obras de madurez, De ludo globi de 1463, a través de la descripción de un juego, el juego de las bolas. El juego que propone consiste en arrojar una bola por una pista en la que se han dibujado nueve círculos concéntricos; el jugador que gana es el que más se aproxima al centro. Para el Cusano, el juego representa el movimiento del alma humana desde su propio reino hasta el reino de la vida, en el cual residen la felicidad y el reposo eternos. Preside el centro del reino Jesucristo, quien, «cuando era semejante a nosotros», movió «la bola de su propia persona» de manera tal que alcanzó el reposo en el centro de vida ${ }^{36}$. De ese modo nos ha dejado su ejemplo, para que hagamos lo que Él hizo y para que nuestra bola siga el recorrido de la suya, aunque es imposible que otra bola repose en el mismo punto en el que descansa la de Cristo.

El movimiento de las bolas se debe al impulso dado libremente por cada jugador. Todas las mentes han sido creadas por Dios pero cada una tiene en sí el principio de su propio movimiento. Cada juego es personal, cada jugador juega su propio movimiento ${ }^{37}$. La mente humana, una vez creada, no es continuamente movida por Dios sino que en ella es creado un movimiento que se mueve a sí mismo; esto es el alma racional ${ }^{38}$. Hay pues, un movimiento, impreso por Dios, por el que el alma se mueve a sí misma y es un

36 Cf. De ludo globi (h IX, $)$ n. 51.

37 Cf. Hans Gerhard Senger, «Dialog über das Kugelspiel» en Handbuch Nikolaus von Kues. Leben und Werk (Darmstadt: WBG, 2014), 255-261; José Luis Caballero Bono, «El globo o el juego de Dios: Deus ludens» en Pensar y conocer a Dios en el siglo XXI, ed. Idelfonso Murillo (Madrid: Diálogos Filosóficos/Publicaciones Claretianas, 2016), 451-457.

38 De ludo globi (h IX) n. 22. «Et licet motus vivificandi animal cesset defici ente sanítate corporis, tamen non cessat motus intellectualis animae humanae, quem sine corpore habet ex excet. Ideo motus ille seipsum intellectualiter movens est in se subsistens et substantialis. Motus enim, qui non est seipsum movens, accidens est. Sed seipsum movens substantia est. Non enim illi accidit motus, cuius natura est motus, uti de natura intellectus, qui non potest esse intellectus sine motu intellectuali, per quem est actu. Ideo intellectualis motus est substantialis seipsum movens». 
movimiento circular y perpetuo. Sin embargo, Nicolás alude allí a otro movimiento que mueve al hombre «todo entero»; esto es, en cuerpo y alma, este movimiento es helicoidal ${ }^{39}$. Aquí pues llama «anima que se mueve a sí misma», lo que en De mente llamó exclusivamente mens o intellectus, y también llama anima al principio de movimiento del cuerpo. Aludiendo a las nociones de sustancia y accidente dice:

Y aunque el movimiento de vivificar el animal cesa cuando la salud del cuerpo es deficiente, no cesa sin embargo el movimiento intelectual del alma humana, que esta tiene y ejerce sin el cuerpo. Porque aquel movimiento que se mueve intelectualmente a sí mismo es subsistente en sí, y es substancial. Pues el movimiento que no se mueve a sí mismo es accidente. Pero el que se mueve a sí mismo es substancia. Pues este movimiento no le acaece a aquello cuya naturaleza es movimiento, como es el caso de la naturaleza del intelecto, el cual no puede ser intelecto sin el movimiento intelectual, por medio del cual está en acto. Porque el movimiento intelectual es substancial y se mueve a sí mismo ${ }^{40}$.

El primer tipo de movimiento mencionado, impreso al cuerpo por la mente mientras esta cumple su función de alma, se detiene cuando el cuerpo muere. En cambio el movimiento circular, por el cual la mente se mueve a sí misma, nunca cesa, este argumento sobre la inmortalidad del alma ya había aparecido en De mente ${ }^{41}$. Este movimiento, dice ahora el cardenal, es «in se subsistens». El vocabulario aristotélico, aquí resignificado, enfatiza un dato importante: el movimiento connatural a la mente no es algo que le acaece, que le adviene de afuera. Ese movimiento es «substancia», existe en sí y no en otro. Quisiera resaltar este punto, la noción de sustancia a la que aquí recurre -como ya lo había hecho en De

39 Cf. Paula Pico Estrada, «Movimientos helicoidal y circular como símbolos de la libertad humana en el diálogo De Ludo Globi de Nicolás de Cusa» en Seminários do Seridò. Solidâo e Libertade, eds. Oscar Federico Bauchwitz, Edrisi Fernández y Cícero Bezerra (Natal: EdufrN, 2014), 351-369.

40 Cf. De ludo globi ( $\left.\mathrm{h} \mathrm{IX}_{1}\right)$ n. 24: «Et licet motus vivificandi animal cesset deficiente sanitate corporis, tamen non cessat motus intellectualis animae humanae, quem sine corpore habet et exercet. Ideo motus ille seipsum intellectualiter movens est in se subsistens et substantialis. Motus enim, qui non est seipsum movens, accidens est. Sed seipsum movens substantia est. Non enim illi accidit motus, cuius natura est motus, uti de natura intellectus, qui non potest esse intellectus sine motu intellectuali, per quem est actu. Ideo intellectualis motus est substantialis seipsum movens.» (Traducción propia). Cf. De mente (h v 2) n. 57. 
mente, cuando habla de «viva substantia»-implica exclusivamente autonomía ontológica, de ninguna manera debe ser entendida como sustrato. La mente no es un «algo» sino puro movimiento intelectual. El intelecto no es una facultad sino la fuerza de intelección. Inteligir es para el intelecto subsistir. Sustancia es aquí solo subsistencia, subsistencia viva y fecunda.

La irreductible unidad de cada mente y su movimiento singularísimo y libre hace que despliegue su propia numeración del mundo. Cada persona tiene un mundo, cada lector despliega un sentido del libro que Dios ha escrito y cuyo sentido último desconoce. Como dice el Cusano en De coniecturis a su amigo Juliano Cesarini, a quien dedica la obra:

Por lo tanto la singularidad todo singulariza, la especialidad especializa, la generalidad generaliza, la universalidad universaliza. Pues todo lo universal, lo general y lo especial en ti, Juliano, «julianiza» como la armonía en el laúd hace lo propio del laúd, en la cítara lo propio de la cítara, y así de los demás. Y tampoco es posible esto en otro como en ti²².

La fórmula podría ser atribuida a cada persona, a su irreductible unidad y a su irreductible mundo asimilado, alteridad de sí mismo, un otro que es despliegue único de sí mismo. De este modo, la novedad cusana no consiste en resaltar la unidad de cada mente sino la singularidad de su despliegue numérico.

\section{Bibliografía}

Beierwaltes, Werner. Identität und Differenz. Frankfurt am Main: Klostermann, 1980.

Beierwaltes, Werner. Platonismus und Idealismus. Frankfurt am Main: Klostermann, 1972.

42 De coni (h. III) n. 89: «Singularitas igitur omnia singularizat, specialitas specializat, generalitas generalizat, universalitas universalizat. Omnia enim universalia, generalia atque specialia in te Iuliano iulianizant, ut harmonia in luto lutinizat, in cithara citharizat, et ita de reliquis. Neque in alio hoc ut in te possibile est». 
Benz, Huber. Individualität und Subjektivität. Interpretationstendenzen in der Cusanus-Forschung und das Selbstverständnis des Nikolaus von Kues. Münster: Aschendorff, 1999.

Bocken, Inigo. «Die Zahl als Gründlage der Bedeutung bei Nikolaus von Kues», Mitteilungen und Forschungsbeiträge der Cusanus-Gesellschaft, Vol. 29 (2005): 201-220.

Caballero Bono, José Luis. «El globo o el juego de Dios: Deus ludens». En Pensar y conocer a Dios en el siglo xxI, editado por Idelfonso Murillo, 451-457. Madrid: Ediciones Diálogos Filosóficos/Publicaciones Claretianas, 2016.

Cassirer, Ernst. Das Erkenntnisproblem in der Philosophie und Wissenschaft der neueren Zeit. Berlín: B. Cassirer, 1906.

D'Amico, Claudia. «Elementos neoplatónicos y herméticos en la concepción del hombre de Nicolás de Cusa». En La concepción del hombre en Nicolás de Cusa. Fuentes, originalidad y diálogo con la modernidad, 81-92. Buenos Aires: Biblos, 2015.

Eisenkopf, Anke. Zahl und Erkenntnis bei Nikolaus von Kues. Regensburg: Roderer-Verlag, 2007.

Flasch, Kurt. «Einleitung». En Opera Omnia, Dietrich von Freiberg, Hamburg: Meiner, 1977, I-LI.

Kremer, Klaus. «Erkennen bei Nikolaus von Kues. Apriorismus Assimilation - Abstraktion». Mitteilungen und Forschungsbeiträge der Cusanus-Gesellschaft, Vol. 13 (1978): 23-57.

Kremer, Klaus, «Grösse und Grenze der menschlichen Vernunft». En Nicholas of Cusa. A Medieval Thinker for the Modern Age, editado por Kazuhiko Yamaki, 5-34. Waseda: Curzón, 2002.

Machetta, Jorge Mario. «Introducción». En Nicolás de Cusa. Un ignorante discurre acerca de la mente. Idiota de mente, edición bilingüe, 9-20. Buenos Aires: Biblos, 2005. 
Nicolai De Cusa. De docta ignorantia. Ediderunt Ernestus Hoffmann et Raymundus Klibansky. Lipsiae: in aedibus Felicis Meiner, 1932 (Opera Omnia Volumen I).

Nicolai De Cusa. De coniecturis. Ediderunt Iosephus Kochæ et Carolus Bormann, Iohanne Gerhardo Senger comite. Hamburgi: in aedibus Felicis Meiner, 1972 (Opera Omnia, Volumen III).

Nicolai De Cusa. Dialogus de ludo globi. Edidit commentariisque illustravit Iohannes Gerhardus Senger. XLII. Hamburgi: in aedibus Felicis Meiner, 1998 (Opera Omnia Volumen IX).

Nicolai De Cusa. De Beryllo. Editionem funditus renovatam atque instauratam curaverunt Iohannes Gerhardus Senger et Carolus Bormann. Hamburgi: in aedibus Felicis Meiner, 1988 (Opera Omnia Volumen XI, Fasciculus 1).

Nicolai De Cusa. Idiota de sapientia - de mente. Editionem post Ludovicum Baur alternam curavit Rentata Steiger, duas appendices adiecit Raymundus Klibansky. Hamburgi: in aedibus Felicis Meiner, 1983 (Opera Omnia Volumen v).

Núñez Poblete, Rodrigo. Metafísica de la singularidad. La noción de singularitas en la filosofía de Nicolás de Cusa. Buenos Aires: Biblos, 2015.

Pico Estrada, Paula. «Movimientos helicoidal y circular como símbolos de la libertad humana en el diálogo De Ludo Globi de Nicolás de Cusa». En Seminários do Seridò. Solidâo e Libertade, editado por Oscar Federico Bauchwitz - Edrisi Fernández - Cícero Bezerra, 351-369. Natal: EDUfRN, 2014.

Rusconi, Cecilia. El uso simbólico de las figuras matemáticas en la metafísica de Nicolás de Cusa (1401-1464). Buenos Aires: Biblos, 2012.

Schwaetzer, Harald. «Nikolaus von Kues als Vordenker der Subjektivität». En Zum Subjektbegriff bei Meister Eckhart und Nikolaus von Kues, eds. Harald Schwaetzer, Marie-Anne Vannier, 67-75. Münster: Aschendorff, 2011. 
Senger, Hans Gerhard. «Dialog über das Kugelspiel». En Handbuch Nikolaus von Kues. Leben und Werk, editado por Marco Brösch - Walter Andreas Euler, 255-226. Darmstadt: WBG, 2014.

Stadler, Michael. «Zum Begriff der mensuratio bei Cusanus. Ein Beitrag zur Ortung der cusanischen Erkenntnislehre». Miscelania Mediaevalia Vol. 16 (1983): 118-131.

Stallmach, Josef. «Die cusanische Erkenntnisauffassung zwischen Realismus und Idealismus». Mitteilungen und Forschungsbeiträge der Cusanus-Gesellschaft Vol. 6 (1967): 50-54.

Volkmann-Schulck, Karl-Heinz. Nikolaus Cusanus. Die Philosophie im Übergang vom Mittelalter zur Neuzeit. Frankfurt am Main: Klostermann, 1957.

Von Bredow, Gerda. «Der Geist als lebendiges Bild gottes (Mens viva dei imago)». Mitteilungen und Forschungsbeiträge der Cusanus-Gesellschaft. Vol.13 (1978): 58-67.

Zeyer, Kirstin. Cusanus in Marburg. Hermann Cohens und Ernst Cassirers produktive Form der Philosophiegeschichtsaneignung, Münster: Aschendorff, 2015. 